Alan T. Nettles' ${ }^{1}$ and Michael J. Douglas ${ }^{2}$

\title{
A Comparison of Quasi-Static Indentation Testing to Low Velocity Impact Testing
}

\author{
Reference: Nettles, A.T. and Douglas, M.J., "A Comparison of Quasi-Static \\ Indentation Testing to Low Velocity Impact Testing", Composite Materials: Testing, \\ Design, and Acceptance Criteria, ASTM STP 1416, A.T. Nettles and A. Zureick, Eds., \\ American Society for Testing and Materials, West Conshohocken, PA, 2002.
}

Abstract: The need for a static test method for modeling low-velocity foreign object impact events to composites would prove to be very beneficial to researchers since much more data can be obtained from a static test than from an impact test. In order to examine if this is feasible, a series of static indentation and low velocity impact tests were carried out and compared. Square specimens of many sizes and thickness were utilized to cover the array of types of low velocity impact events. Laminates with a $\pi / 4$ stacking sequence were employed since this is by the most common type of engineering laminate. Three distinct flexural rigidities under two different boundary conditions were tested in order to obtain damage due to large deflections, contact stresses and both to examine if the static indentation-impact comparisons are valid under the spectrum of damage modes that can be experienced. Comparisons between static indentation and low velocity impact tests were based on the maximum applied transverse load. The dependent parameters examined included dent depth, back surface crack length, delamination area and to a limited extent, load-deflection behavior. Results showed that no distinct differences could be seen between the static indentation tests and the low velocity impact tests, indicating that static indentation can be used to represent a low velocity impact event.

Keywords: composites, static indentation, impact, transverse loading rate

Low velocity impact events are expected to occur during the manufacturing and service life of composite parts and/or structures. Foreign body impact can occur during manufacturing, routine maintenance or use of a laminated composite part. This has led to an abundance of research on low-velocity impact damage to laminated composite plater Typically, laminated plates are impacted by a "drop weight" method. This method usually consists of an instrumented striker (tup) that is secured to a carriage that falls along guideposts and collides with the plate. After an impact event has been performed, ultrasonic $\mathrm{C}$-scans, $\mathrm{x}$-radiography and cross sectional photo-microscopy are some of the common techniques used to document the damage area. Post impact strength testing (mostly compression) is often performed to evaluate a material's or structure's damage tolerance.

It would be very beneficial to simulate an impact event using a "quasi-static" loading test. By using this test, damage initiation and propagation can be more easily detected,

\footnotetext{
${ }^{1}$ Composite Materials Engineer, NASA Marshall Space Flight Center, MSFC, AL 35812

2 Design Engineer, International Construction Equipment, Matthews, NC 28104
} 
deflection can be directly measured with great accuracy and maximum transverse force can be better controlled. Thus it is the focus of the work in this study to examine if drop weight impact tests and quasi-static loading tests give the same size, shape and location of damage for a given maximum transverse load.

In the present study, all tests were conducted on laminated plates made from an intermediate modulus carbon fiber with a toughened epoxy resin in prepreg form. The plates tested were quasi-isotropic with a stacking sequence of $[+45,90,-45,0]_{\mathrm{ns}}$. With $\mathrm{n}$ equal to $1,2,4$, or 6 .

\section{Previous Work}

\section{Background}

The need for a static (or more commonly referred to as quasi-static) test method for modeling low-velocity foreign object impact events would prove to be very beneficial to researchers since much more data can be obtained from a quasi-static test than from an impact test. ASTM Standard D6264-98 has been proposed for transverse quasi-static loading of composite laminates, although the standard stops short of claiming to represent low-velocity impacts. Since a "low-velocity" impact event last approximately 6-10 milliseconds there is debate as to whether or not a quasi-static indentation test truly represents a low velocity impact event.

The first order of business is to determine whether or not an impact event is considered "low-velocity" and can thus be subject to further analysis as a quasi-static event. It has been clearly shown that projectile-type impacts in the ballistic range are governed by dynamic events and therefore could never be represented by a quasi-static test $[1,2,3]$. Some research efforts have been focused on defining the bound between "low-velocity" and "dynamic" impact events. One study suggested that the impactor to target frequency ratio governs the type of event with a low (much less than unity) ratio implying a quasi-static event [4]. A simpler method was obtained by Swanson [5] in which a rule has been established that if the impactor mass is more than ten times the "lumped mass" of the target, then the impact event will be quasi-static in nature. The "lumped mass" is a function of the target shape and boundary conditions but is generally about one-half the mass of the entire target. However, for most practical purposes it is fairly clear if an impact event is "low-velocity" or not. High-velocity/large mass impacts of are little concern since the part will be so heavily damaged by such an event that an analysis is not needed and conversely a low velocity/low mass impact is of little concern since no damage will form.

Once an impact event is deemed to be "low-velocity" the question remains as to whether or not a static indentation test can be performed that will duplicate certain aspects of the impact. Some of these aspects include permanent indentation, maximum displacement and most importantly, amount and type of damage formed. All of these parameters must be compared against an independent variable that will be common to both tests. It has been suggested that this independent variable be the maximum transverse load $[3,6,7]$. 
Permanent indentation after an impact or quasi-static loading test has been examine in a few studies $[8,9,10]$. The one common feature to all of these studies is the large amount of scatter in indentation depth data to the point of rendering this measurement useless. Nevertheless, it was decided to examine this parameter in this study to see how much scatter would exist.

For load-deflection correlation it is imperative to have an instrumented impact apparatus. The interpretation of the signals has been greatly simplified with the use of commercially available systems that filter the load signals to reduce unwanted noise. Care must be taken to ensure that the filter being used does not mask important load events. A complete analysis of instrumented impact testing is beyond the scope of this study, but two excellent references are noted for the reader $[11,12]$.

The amount of damaged formed by an impact event can be measured in a number of ways. Destructively, the impacted specimen can be sectioned and examined under high magnification, or a residual property can be measured (termed "damage tolerance"). Nondestructively, ultrasonic or $x$-radiography can give a planar indication of the type and extent of damage. Ultimately the amount of damage formed by an impact event is of the most concern to the engineer investigating such an occurrence and since the impacted part may still be useable, non-destructive techniques are preferred. Thus the major portion of this study will deal with the resulting damage as detected via non-destructive evaluation, and whether or not the damage formed for a given transverse load is similar in low-velocity impact and quasi-static testing.

\section{Impact versus Quasi-Static Testing}

Several studies $[3,6,8,13,14]$ show a similarity between quasi-static indentation and drop weight impact testing. While other studies, $[7,15,16]$ have shown a limit to the applicability of using quasi-static indentation to represent impact events. It must be noted that there are many variables involved in these tests such as boundary conditions, specimen size, specimen thickness, stacking sequence, impactor size, impactor shape and type of fiber/resin system. The amount of impact damage formed in a laminated composite has been shown to be very sensitive to stacking sequence, regardless of thickness [17]. As plies are grouped together, larger areas of delaminations tend to form. It has been conventional wisdom in the composites industry to disperse the ply orientations in order to increase damage resistance. For example, a stacking sequence of $[+45,0,-45,90]_{2 \mathrm{~S}}$ is preferable to one of $\left[+45_{2}, 0_{2},-45_{2}, 90_{2}\right]_{\mathrm{S}}$ in order to increase the damage resistance of the laminate.

\section{Experimental Procedure}

The intent of this study was to compare quasi-static indentation testing to drop weight impact testing based on the maximum transverse load. In order to insure a complete analysis of the two events, the testing was divided into two different categories based on the boundary conditions. These two categories were then subdivided into three additional groups based on the composite laminate's transverse (flexural) stiffness. To insure the repeatability of the experimental procedure each impact test was performed on approximately four different specimens, while the quasi-static indentation tests were 
performed on two different specimens. Because of the inherent scatter in data of the dr weight impact testing, it was decided that portion needed to be repeated numerously. However, the repeatability became so constant during the latter stages of the testing that the number of impacted specimens for repeatability assurance was reduced.

The rate of the quasi-static indentation test was also investigated to find if there was any time dependency involved in quasi-static indentation testing. The two rates used were $0.02 \mathrm{~mm} / \mathrm{s}$ and $0.42 \mathrm{~mm} / \mathrm{s}$.

\section{Boundary Conditions}

The main two categories of test were depended on the boundary conditions. Specimens were either clamped on all four edges or simply supported (free) on all four edges. This was done to determine if the boundary conditions would have a major influence on the damage introduced due to impact or quasi-static loading.

To perform the simply supported test, the specimens were placed on machined plates made from a $5.08 \mathrm{~cm}$ thick aluminum plate with an outside square dimension of 40.64 $\mathrm{cm}$. A total of four plates were made with a square cutout in the center. These cutouts had sizes of $5.08 \mathrm{~cm}, 10.16 \mathrm{~cm}, 15.24 \mathrm{~cm}$, and $30.48 \mathrm{~cm}$. This was done to explore the effects of the transverse flexural properties of the composite panels.

In order to perform the test with clamped boundary conditions the plates had a series of $0.64 \mathrm{~cm}$ holes drilled and tapped into the aluminum plate $3.81 \mathrm{~cm}$ from the edge of the opening. The holes were spaced $2.54 \mathrm{~cm}$ on center. A $1.27 \mathrm{~cm}$ thick steel plate was machined with holes placed in the same location as those in the aluminum plate. The laminated composite panel to be tested was placed between the aluminum plate and the steel plate. Socket head bolts were then used to secure the specimen and a uniform torque of $5.65 \mathrm{~N} \cdot \mathrm{m}$ was applied to each bolt.

\section{Flexural Rigidity of Specimens}

The three subgroups of tests involved the transverse stiffness of the composite plates. The stiffness was a function of the ratio of the support opening size versus the laminate thickness. The specimens were divided into three categories under this assumption: Flexible, Medium and Stiff with ratios of 150,50 and 25 respectively. During an impact event this transverse stiffness characteristic changes the mode of damage. For stiff laminates the contact forces caused the majority of damage which was more confined to the side of the plate hit by the impactor. For flexible laminates the damage was most severe on the side opposite the impact site. This is characteristic of the materials used in advanced composites.

\section{Specimen Preparation}

The quasi-isotropic laminated panels were layed-up by hand from the carbon/epoxy prepreg material, placed in a vacuum bag and autoclave cured using the manufacturer's cure cycle. The panels were fabricated into $61.0 \times 91.4 \mathrm{~cm}$ plates. In order to obtain a large variety of flexural stiffnesses of laminates the following four thicknesses (based on number of plies) were used, $8,16,32$, and 48-ply. The panels were fabricated utilizing 
the quasi-isotropic $\pi / 4$ stacking sequence of $[+45,90,-45,0]_{\mathrm{ns}}$, where ' $n$,' was givent the value of $1,2,4$, or 6 respectfully in order to obtain the desired number of plies.

\section{Impact Testing Procedure}

The impact testing was performed using a drop-weight impact tester. The specimens were placed on the platens with the desired opening size. Table 1 lists the opening (or span) size used depending on the laminate plate thickness. This divided the tests up into the proper flexural rigidity ratios being examined.

Table 1 - Opening and laminate thickness ratios.

Number of Plies, Laminate Thickness (mm), Opening or Span Size (mm)

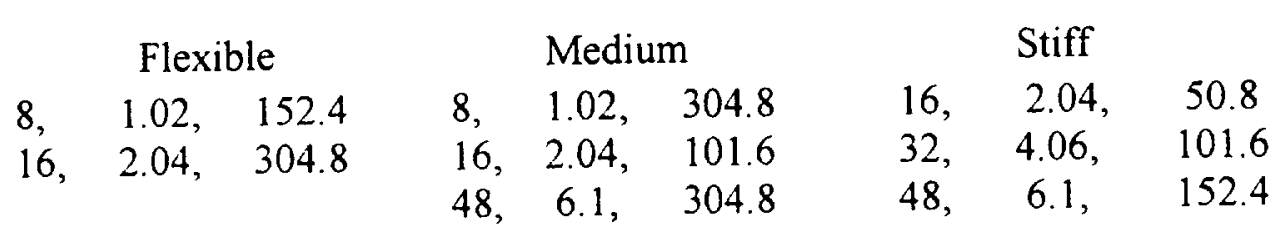

Specimens were then impacted with a hemispherical tipped steel tup. The drop height and mass of the impactor were adjusted to give the desired damage level. The damage level desired was very little visual damage to the top of the specimen while achieving a measurable crack on the bottom surface. This level of damage was chosen since the onset of visual damage is such a critical state for an impact event. If penetration is allowed, boundary conditions and rate effects will not be as noticeable and if too low of an impact level is used, damage may not from at all. Table 2 lists the height, max load, boundary condition and mass for each subgroup that was finally chosen for impact testing.

Table 2-Maximum load and drop height for the impact specimens tested.

Number of Plies, Mass (kg), Impact Force (N), Drop Height $(\mathrm{cm})$, Boundary Condition

Flexible

$8,2.4,1930,30.5$, Clamped $16,2.4,7108,121.9$, Clamped

$8,2.4,1873,44,5$, Free $16,2.4,5400,148.6$, Free
Medium

$8,2.4,1036,12.7$, Clamped $16,2.4,3728,35.6$, Clamped $48,13.3,26823,119.4$, Clamped $8,2.4,974, \quad 5.7, \quad$ Free $16,2.4,3728,49.5$, Free $48,13.3,23562,119.4$, Free
Stiff

$16,2.4,3100,33.0$, Clamped $32,2.4,7268,71.1$, Clamped $48,13.3,23100,63.5$, Clamped $16,2.4,2922,52.7$, Free $32,2.4,9853,124.5$, Free $48,13.3,22121,63.2$, Free

\section{Quasi-Static Indentation Testing Procedure}

Once the impact testing was completed, the maximum impact force obtained for each of the different subgroups (listed in Table 2) was used as the independent variable for the quasi-static indentation testing. Figure 1 shows the test fixture used for all of the quasistatic indentation test performed. The platen rested on top of the $5.08 \mathrm{~cm}$ thick aluminum uprights and could be removed without taking the fixture out of the test frame. The aluminum uprights were bolted to a $5.08 \mathrm{~cm}$ thick steel plate. The indentor was placed in 
the upper crosshead of the servo hydraulic load frame. In a limited number of tests, transverse deflection of the center point of the laminate, directly under the hemispherical tup, was measured using a linear voltage displacement transducer (LVDT). Figure 1

shows the location of the LVDT. The tests were run in stroke control at load rates of 0.02 and $0.42 \mathrm{~mm} / \mathrm{s}$.

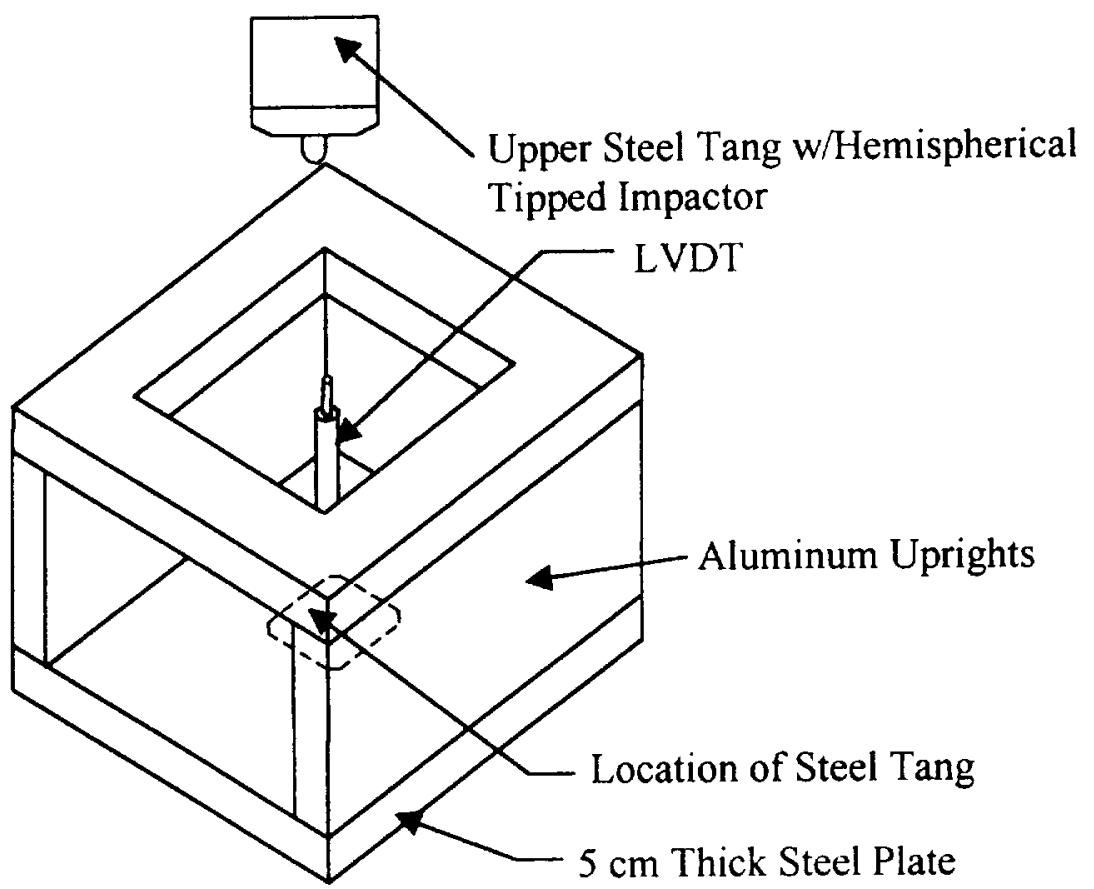

Figure 1 - Test fixture for quasi-static indentation testing.

Non-Destructive Analysis

Once the impact and quasi-static testing were completed the specimens underwent three types of non-destructive analysis to document internal and external damage. These consisted of measuring dent depth on the impacted surface, crack lengths on the nonimpacted surface and internal damage as determined from x-radiography.

After the specimens were impacted or subjected to quasi-static indentation testing, they were set aside for at least 24 hours so that the resulting dent would have time to relax to its equilibrium state. Any visible cracks on the non-impacted surface of the specimens were measured.

After all dent depths and crack lengths were measured the specimens were then subjected to radiographic techniques to document the internal damage. The specimens were soaked on both sides with a zinc iodide penetrant solution for 24 hours and then $x-$ rayed using a Faxitron ${ }^{T M} \mathrm{X}$-ray machine. A piece of photgraphic film was placed directly 
under the specimen to capture the image of the internal planar dar age in the form of a negative. From the negatives positives were made so that the planner damage area could be calculated. This was accomplished by superimposing a grid of $4 \mathrm{~mm}^{2}$ squares over the picture and then counting squares that were within the damage area. Figure 2 illustrates the process used.

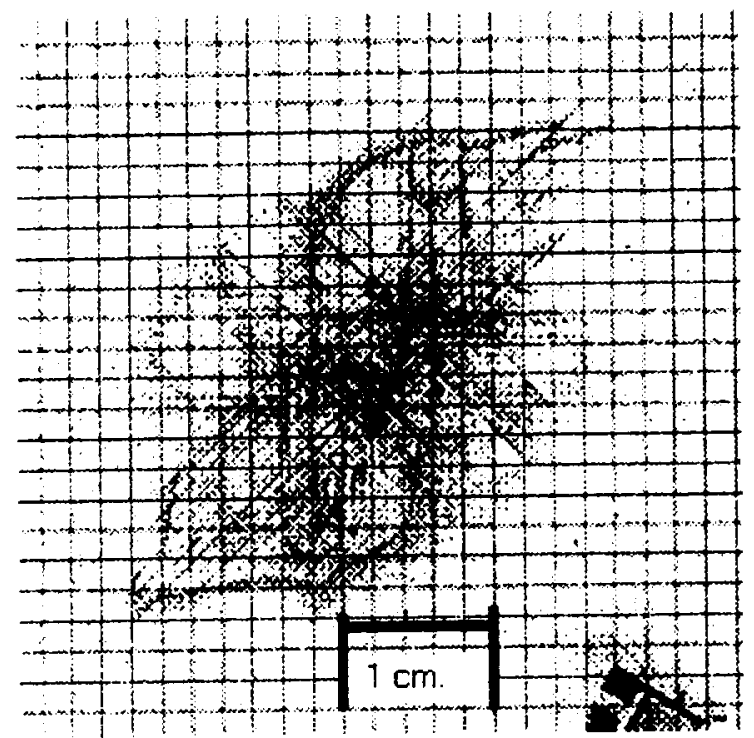

Figure $2-X$-ray of impact specimen with $+\mathrm{mm}^{2}$ grid superimposed.

It should be noted that this is only a planar calculation and does not take into consideration the thickness of the specimen. The planar area of delamination was the most important variable used in this study and was the main factor in determining if an impact event can be represented by a quasi-static indentation test.

\section{Results and Discussion}

\section{Introduction}

Since the main purpose of the research being presented was to establish if quasi-static indentation testing was a true representation of a low velocity impact event, this section will address this issue by comparing the experimental results obtained. Table 2 lists the maximum transverse loads used for comparison between impact and quasi-static testing. Once the specimens were tested, comparisons were made on the following; dent depth, crack length, delamination area, and to a limited extent, load-deflection data.

\section{Dent Depth}

Plots of dent depth versus maximum transverse load demonstrate the vast amount of scatter that exists in the dent depth measurements thus making trends difficult to identify. There appears to be no difference between the dent depth of an impacted specimen versus that of a quasi-statically loaded one. Two examples of these types of plots are shown in figure 3 . The clamped and free boundary conditions are shown together for comparison. 

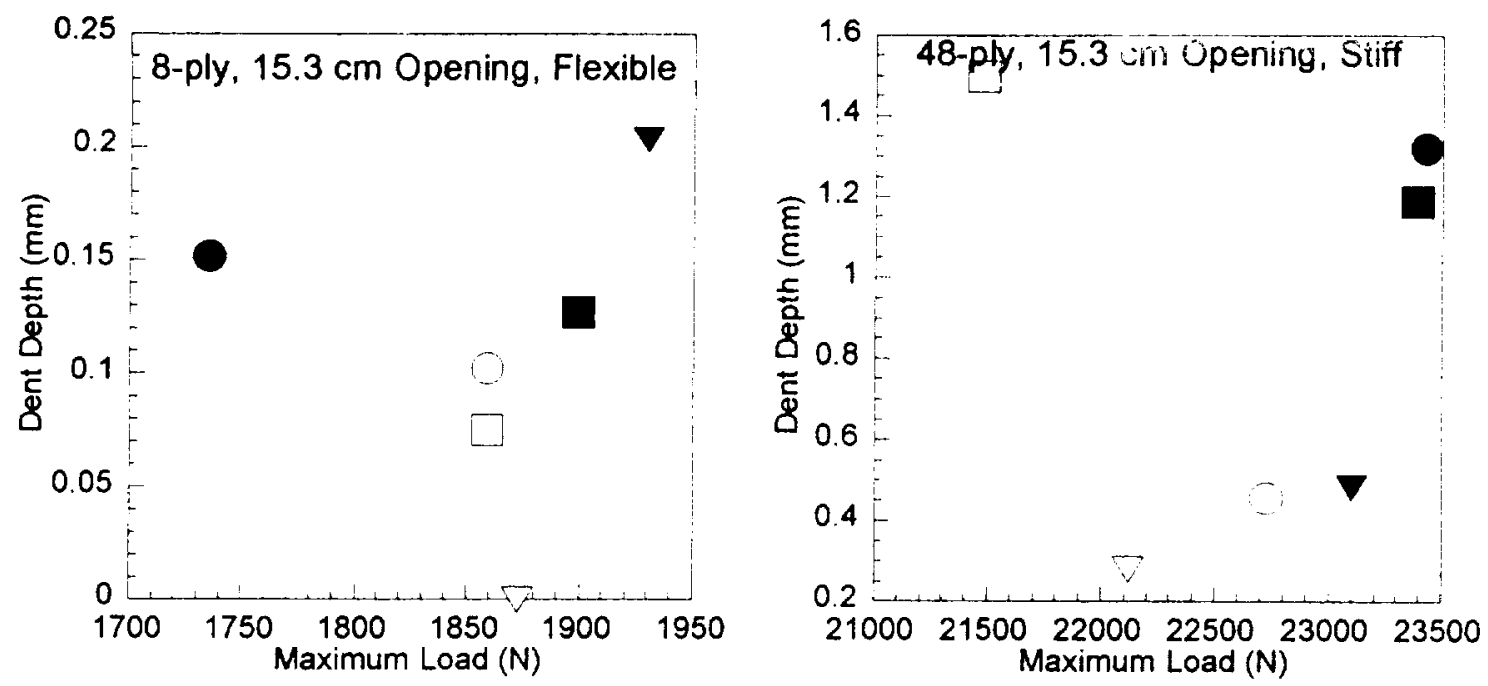

Figure 3 -Plots of dent depth versus maximum transverse load. $\nabla$-Impact; O- $1.27 \mathrm{~mm} / \mathrm{sec}$ Static; $\square-25.4 \mathrm{~mm} / \mathrm{sec}$ Static Open $=$ Free $;$ Filled $=$ Clamped

\section{Crack Length}

The length of the crack, or split, on the opposite side from the impact site also demonstrated a large amount of scatter. For the specimens that were impacted, the split length was longer than an equivalently load quasi-static specimen. Two examples of data are presented like they were for dent depth and are shown in figure 4.
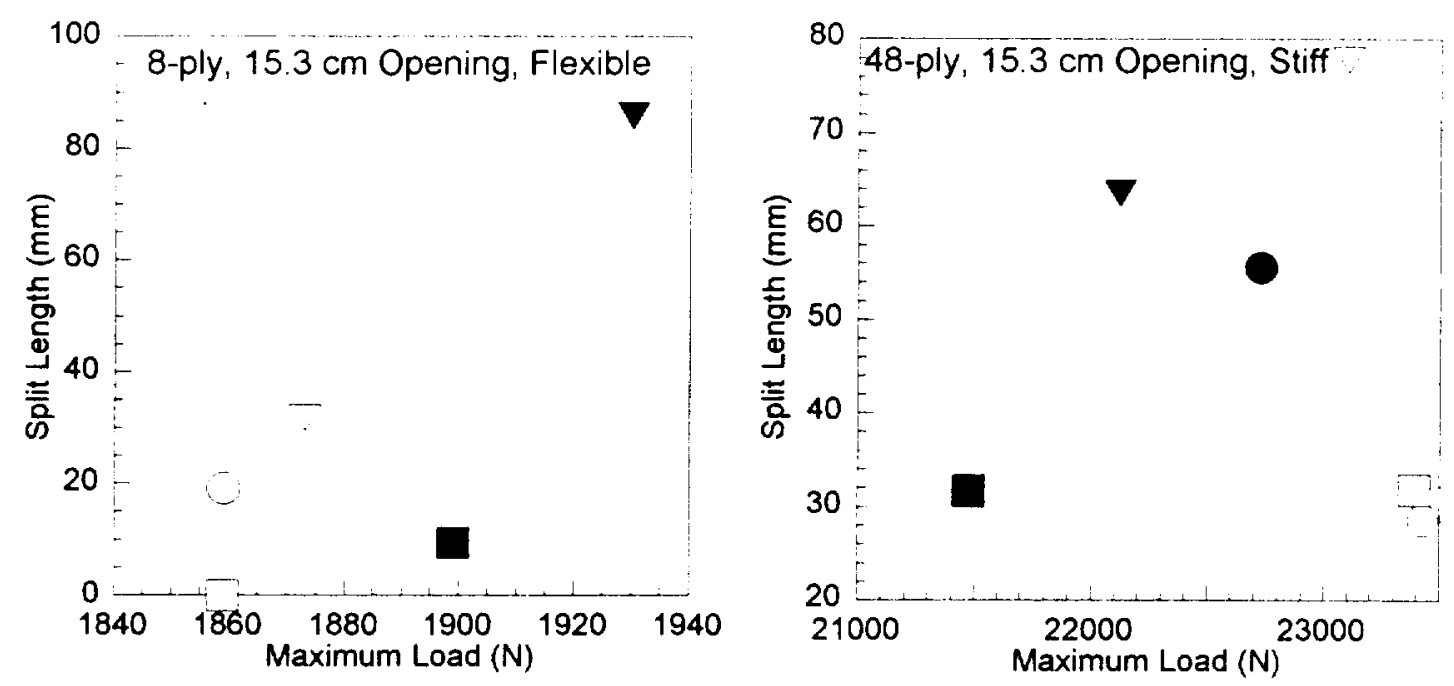

Figure 4-Plots of split length versus maximum transverse load. $\nabla$-Impact; O- $1.27 \mathrm{~mm}$ sec Static; $\square-25.4 \mathrm{~mm}$ sec Static Open $=$ Free; Filled $=$ Clamped 


\section{Delamination Area}

Figures 5-12 present delamination area as a function of applied transverse load for both low-velocity impacts and quasi-static loads of two rates. Each figure contains data for both clamped and simply supported (free) specimens

$\nabla$-Impact; $\bigcirc-1.27 \mathrm{~mm}$ sec Static; $\square-25.4 \mathrm{~mm}$ sec Static Open $=$ Free $;$ Filled $=$ Clamped

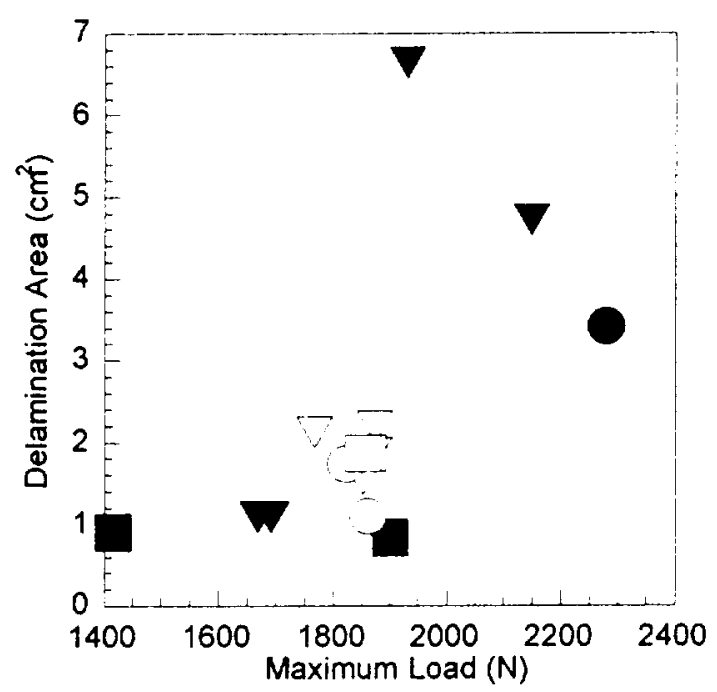

Figure 5 - Delamination area versus transverse load, 8-ply specimen over $152.4 \mathrm{~mm}$ opening, flexible.

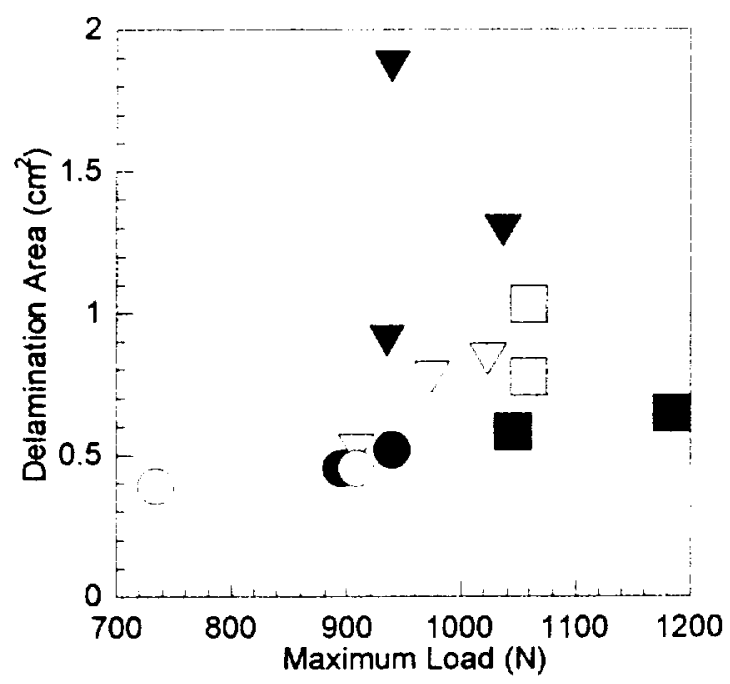

Figure 7 - Delamination area versus transverse load, 8 ply specimen over $50.8 \mathrm{~mm}$ opening, flexible.

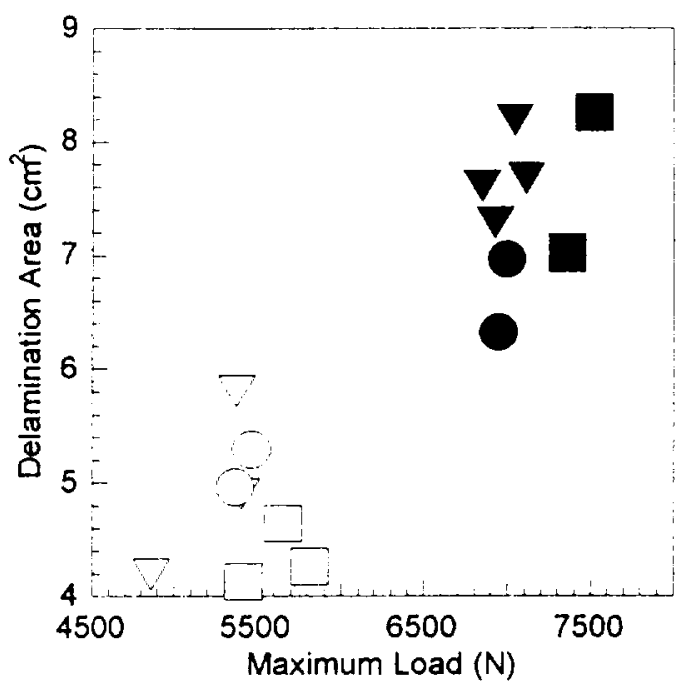

Figure 6 - Delamination area versus iransverse load, 16-ply specimen over $304.8 \mathrm{~mm}$ opening, flexible.

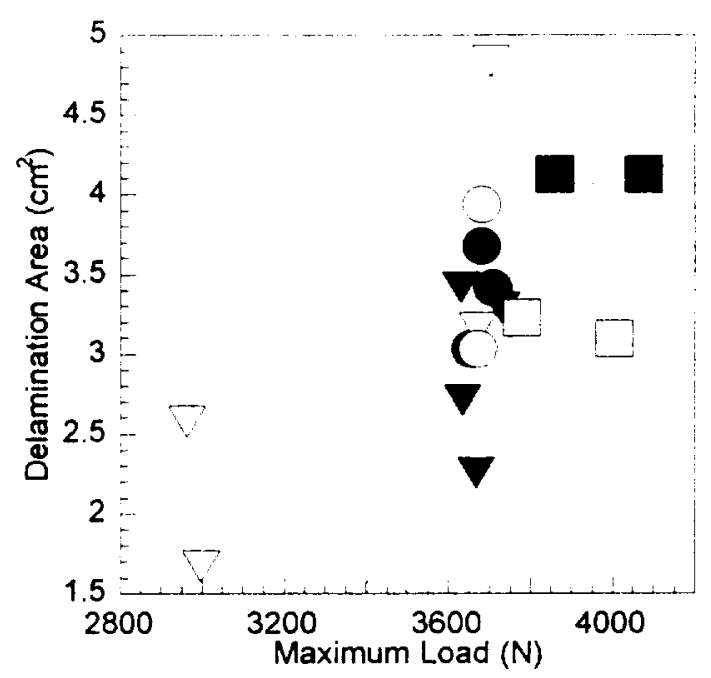

Figure 8 - Delamination area versus transverse load. 16 ply specimen over $101.6 \mathrm{~mm}$ opening, medium. 


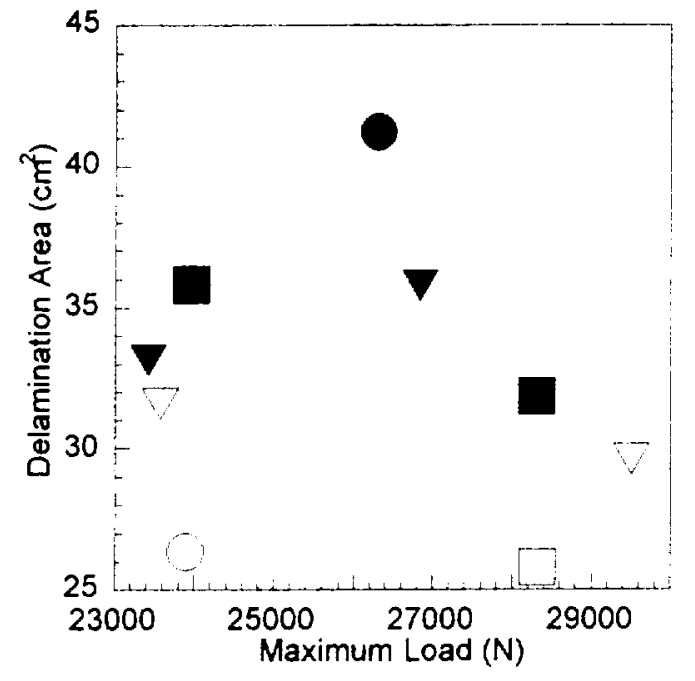

Figure 9 - Delamination area versus transverse load, 48-ply specimen over $304.8 \mathrm{~mm}$ opening, medium.

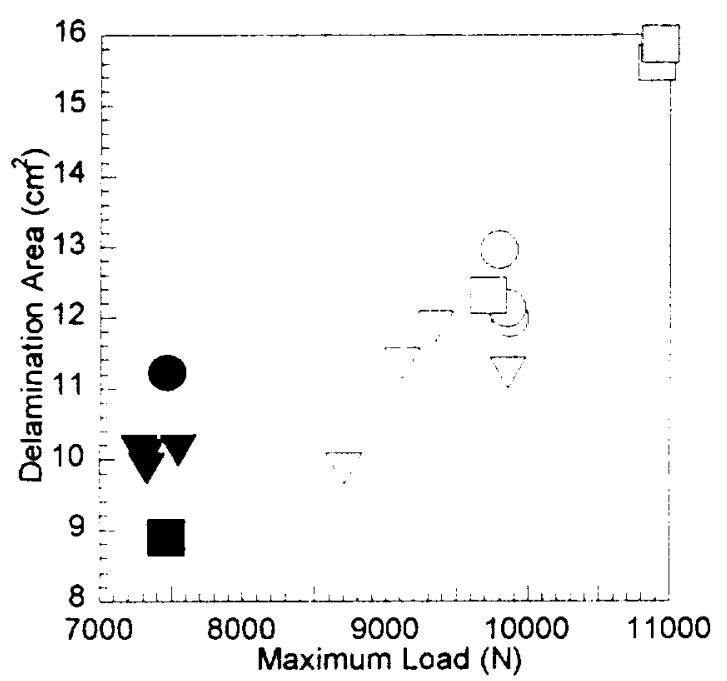

Figure 11 - Delamination area versus transverse load, 32-ply specimen over $101.6 \mathrm{~mm}$ opening, stiff.

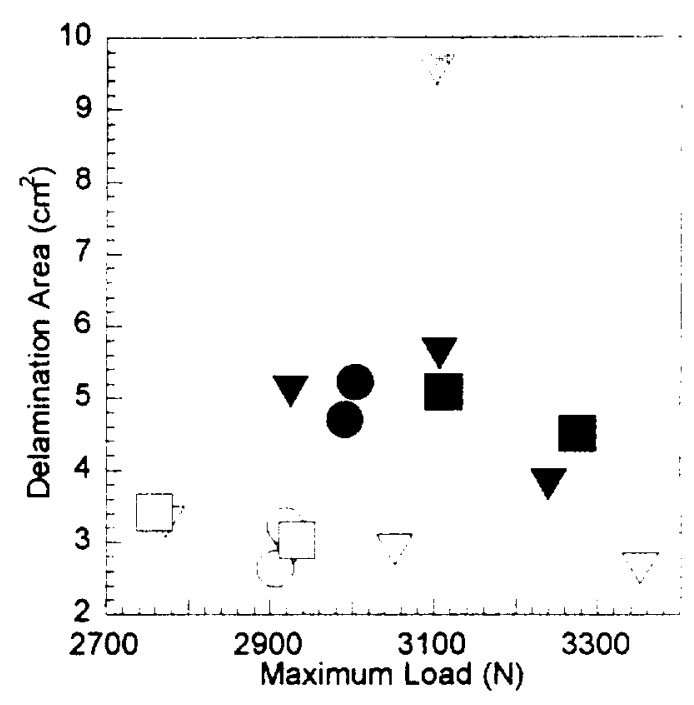

Figure 10 - Delamination area versus transverse load, 16-ply specimen over $50.8 \mathrm{~mm}$ opening, stiff.

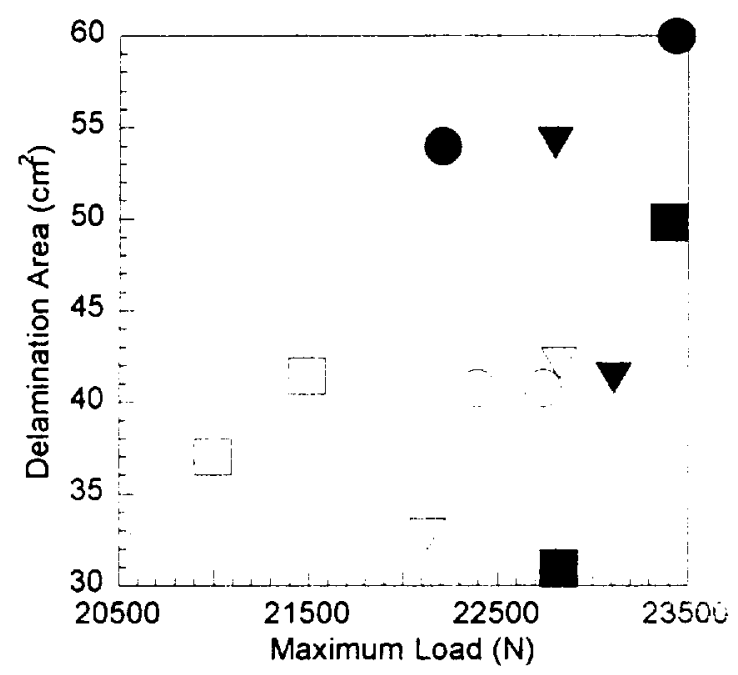

Figure 12 - Delamination area versus transverse load, 48-ply specir over $152.4 \mathrm{~mm}$ opening, stiff.

Figures 5 and 6 present data for the case of "flexible" laminates (support/thickness ratio of 150). Figures 7-9 present data for the case of "medium" laminates (support/thickness ratio of 50). Figures 10-12 present data for the case of "stiff" laminates (support/thickness ratio of 25 ).

For the "flexible" laminates there is no distinct difference between the impacted specimens and the ones tested quasi-statically at either rate. The effects of the boundary conditions show no difference for the 8 ply specimens supported over the $152.4 \mathrm{~mm}$ opening whereas a distinct difference is seen for the 16 ply specimens supported over the $304.8 \mathrm{~cm}$ opening. This difference is due to the clamped specimens being loaded to a higher level resulting in a larger delamination area. 
The "medium" specimens have no distinct trends between boundary conditions or: of loading. The impact test results fall in well with the static indentation tests in figures 7 , 8 and 9.

Figures 10, 11 and 12 represent the opposite extreme from the "flexible" specimens in that the contact damage dominates. Again there is no discernable difference between impact and static indentation results. In figure 10, the simply supported specimens show slightly less damage for the same magnitude of maximum load than the clamped specimens, however this difference is slight.

Overall the low velocity impact tests can be represented by static indentation testing at rates of .02 and $0.42 \mathrm{~mm} / \mathrm{sec}$, regardless of specimen rigidity and boundary conditions. There is enough inherent scatter in both types of tests that all data fall within this scatter.

It must be kept in mind that these results are only valid for laminates of the $\pi / 4$ type and laminates with different lay-ups or clumped plies may yield different results.

\section{Load-Deflection Data}

As another check of the validity of using static indentation tests to represent impact tests, a comparison of the load/deflection data can be made.

Figures 13-18 show static indentation load/deflection data superimposed over impact load/deflection data. A circle represents the static data, while the impact data are represented by a triangle. Static load/deflection data was not available for all of the static tests since a faulty LVDT was used, thus only the valid data are presented.

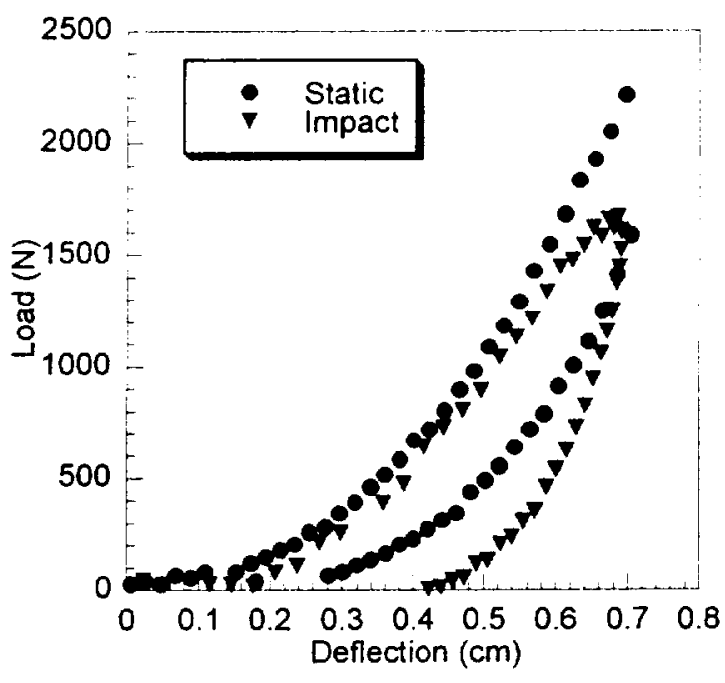

Figure 13 - Static and Impact LoadDeflection Data for 8-ply Specimen Over 152.4-mm Opening; Clamped.

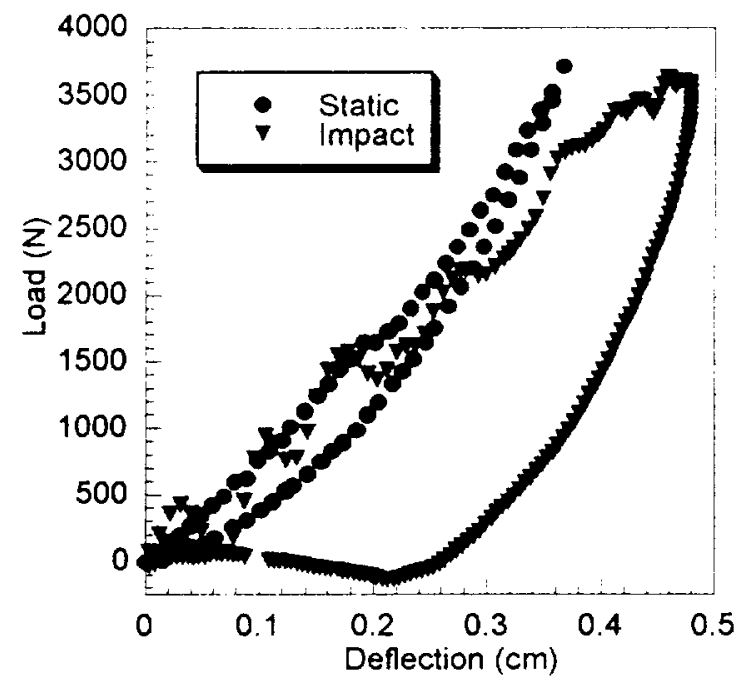

Figure 14 - Static and Impact LoadDeflection Data for 16-ply Specimen Over 101.6-mm Opening; Clamped. 


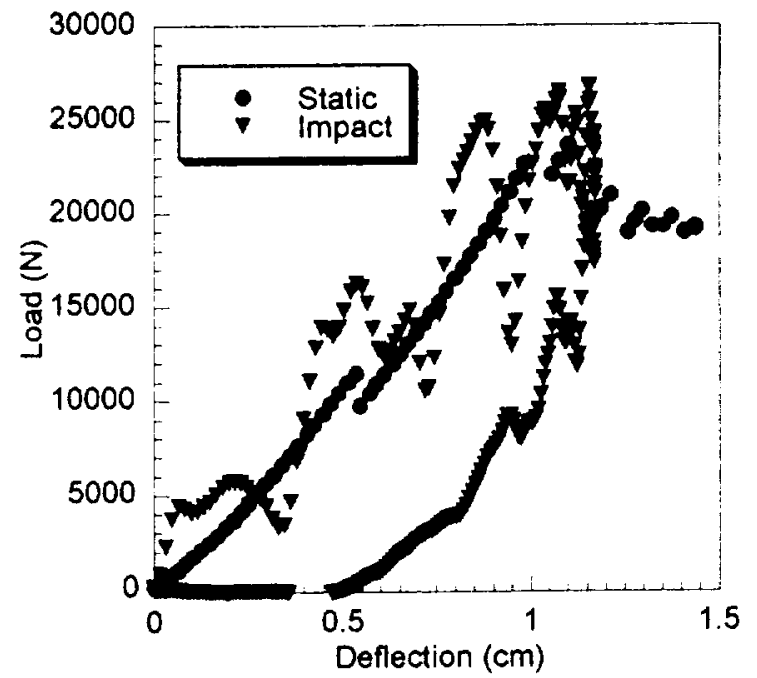

Figure 15 - Static and Impact LoadDeflection Data for 48-ply Specimen Over $304.8 \mathrm{~mm}$ Opening; Clamped.

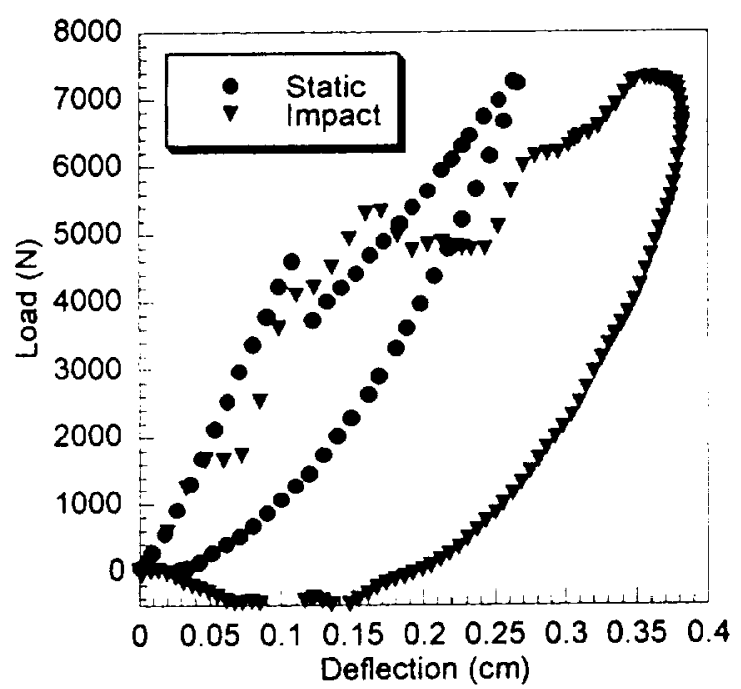

Figure 17 - Static and Impact LoadDeflection Data for 32-ply Specimen Over $101.6 \mathrm{~mm}$ Opening; Clamped.

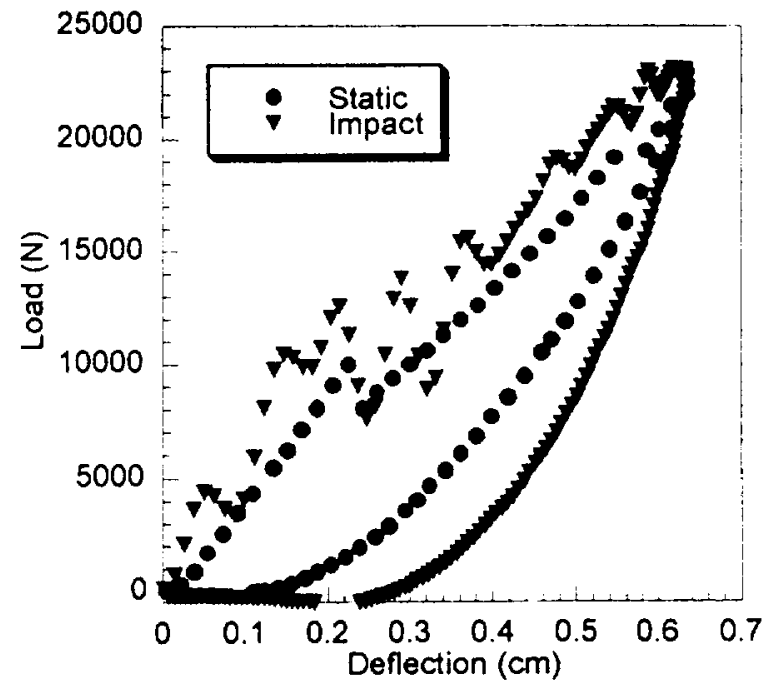

Figure 16 - Static and Impact LoadDeflection Data for 48-ply Specimen Over 152.t-mm Opening; Clamped.

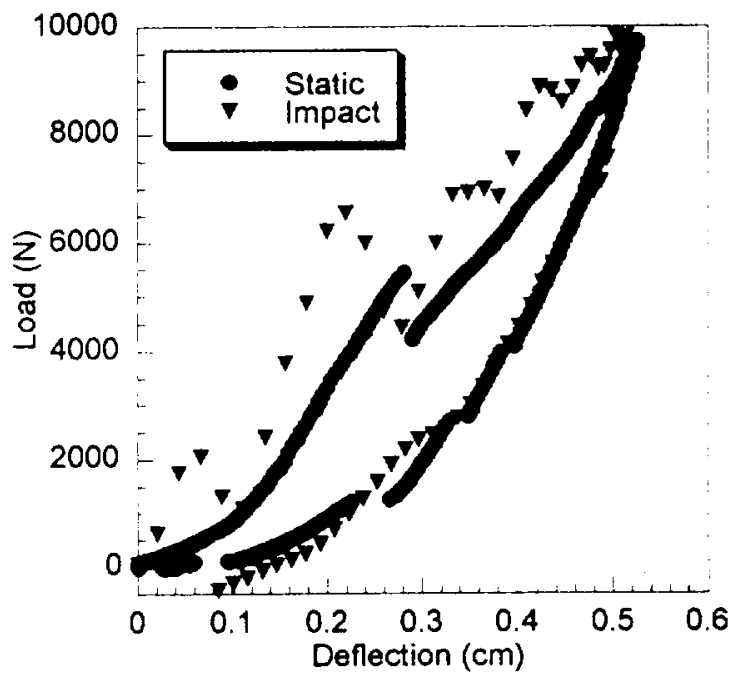

Figure 18 - Static and Impact LoadDeflection Data for 32-ply Specimen Over $101.6 \mathrm{~mm}$ Opening; Free.

On the loading portion of the curves, the data agree well, except for the undulations in load seen on the specimens which experience a relatively high load. However, for the unloading portion of the curves the impact data usually indicate that more energy was lost during the event since there is a much larger hysteresis in the impact curves. However, from the delamination area data it was anticipated that the energy lost should be about the 
same. It has always been suspected that most of the energy lost in this type of impact testing is lost due to vibrations within the impact apparatus, not in damage to the specimen. When the falling crosshead and tup impact the composite plate, the head will tend to rebound at an angle that is not parallel to the guideposts. Thus a sideways force is exerted on the guideposts, which causes them to vibrate and interfere with the "natural" rebound of the impactor had the guideposts not been a factor.

Figures 14, 16 and 17 represent this erroneous "loss of energy" data quite well. Figure 13 is a flexible specimen and the loading portions of the curves agree well for both the static and impact cases. However, for unloading, the impact data shows a larger deflection than the static data for a given load on the rebound.

Figure 15 does not have unloading data for the static case, but the loading portions of the curves agree quite well. Figure 15 is unique in that during the static indentation test, the maximum load as determined from the impact tests could not be reached since the impactor began penetrating through the plate before this load was obtained. This is a result of selecting the onset of "easily" visible damage as the point at which the impact tests were carried out. For a relatively thick plate, the load at which a back surface split appears is not much below the load needed to begin penetration since so much volume of material needs to be damaged to cause the back surface split.

\section{Conclusions}

The following are the major conclusions of this study:

1. Static indentation tests can be used to represent low velocity impact events when the damage is compared by maximum transverse force. This is true of plates that experience flexural type damage, contact type damage and a combination of the two. Lay-ups other than of the $\pi / 4$ type may not yield these same results.

2. Much non-linear behavior is observed in the load-deflection curves for flexible laminates. As the laminate becomes stiffer, more linearity is seen and a distinct drop in load due to delamination becomes more pronounced.

3. Load-deflection plots of static indentation and low velocity impact are similar for the loading portion of the curve. The impact plots tend to show more energy is lost.

4. Dent depth results produce a great deal of scatter, which makes any conclusions concerning this parameter difficult.

5. The only difference in damage between statically loaded and impacted specimens may be the split length that forms on the underside of the specimen, although this difference is small and does not seem to effect the delamination area.

\section{References}

[1]. Cantwell, W.J. and Morton, J., "Comparison of the Low and High Velocity Impact Response of CFRP," Composites, Vol. 20, No. 6, November 1989, pp. 545-551. 
[2]. Wu, E. and Chang, L., "Loading Rate Effect on Woven Glass Laminated Plates by Penetration Force," Journal of Composite Materials, Vol. 32, No. 8, 1998, pp. 702-721.

[3]. Jackson, W.C. and Poe, C.C., "The Use of Impact Force as a Scale Parameter for the Impact Response of Composite Laminates," NASA Technical Memorandum 104189, January, 1992.

[4]. Bucinell, R.B., Nuismer, R.J. and Koury, J.L., "Response of Composite Plates to Quasi-Static Impact Events," Composite Materials: Fatigue and Fracture (Third Volume), ASTM STP 1110, American Society for Testing and Materials, West Conshohocken, PA, 1991, pp. 528-549.

[5]. Swanson, S.R., "Limits of Quasi-Static Solutions in Impact of Composite Structures," Composites Engineering, Vol. 2, No. 4, pp. 261-267.

[6]. Kwon, Y.S. and Sankar, B.V., "Indentation-Flexure and Low-Velocity Impact Damage in Graphite Epoxy Laminates," Journal of Composites Technology and Research, Vol. 15, No. 2, Summer 1993, pp. 101-111.

[7]. Lagace, P.A., Williamson, J.E., Wilson Tsang, P.H., Wolf, E. and Thomas, S., "A Preliminary Proposition for a Test Method to Measure (Impact) Damage

Resistance," Journal of reinforced Plastics and Composites, Vol. 12, May 1993, pp. 584-601.

[8]. Kaczmarek, H. and Maison, S., "Comparative Ultrasonic Analysis of Damage in CFRP Under Static Indentation and Low-Velocity Impact," Composites Science and Technology, Vol. 51, 1994, pp. 11-26.

[9]. Wardle, B.L. and Lagace, P.A., "On the Use of Dent Depth as an Impact Damage, Metric for Thin Composite Structures," Journal of Reinforced Plastics and Composites, Vol. 16, No. 12, 1997, pp. 1093-1110.

[10]. Nettles, A.T., Douglas, M.J. and Estes, E.E., "Scaling Effects in Carbon/Epoxy Laminates Under Transverse Quasi-Static Loading," NASA Technical Memorandum 209103, March 1999.

[11]. Cheresh, M.C. and McMichael, S., "Instrumented Impact Test Data Interpretation," Instrumented Impact Testing of Plastics and Composite Mater. ASTM STP 936, American Society for Testing and Materials, West Conshohocken, PA, 1987, pp. 9-23.

[12]. Found, M.S., Howard, I.C. and Paran, A.P., "Interpretation of Signals from Dropweight Impact Tests," Composite Structures, Vol. 42, 1998, pp. 353-363.

[13]. Lee, S.M. and Zahuta, P., "Instrumented Impact and Static Indentation of Composites," Journal of Composite Materials, Vol. 25, Feb. 1991, pp. 204-222.

[14]. Sjoblom, P.O., Hartness, T.J. and Cordell, T.M., "On Low-Velocity Impact Testing of Composite Materials," Journal of Composite Materials, Vol. 22, Jan. 1988, pp. 30-52.

[15]. Elber, W., "Failure Mechanics in Low-Velocity Impacts on Thin Composite Plates," NASA Technical Paper 2152, 1983.

[16]. Highsmith, A.L., "A Study of the Use of Contact Loading to Simulate Low Velocity Impact," NASA Contractor Report 97-206121, Jan. 1997.

[17]. Abrate, S., "Impact on Laminated Composites: Recent Advances," Applied Mechanics Review, Vol. 47, No. 11, 1994, pp. 517-544. 